\title{
An anomalous peak observed in the electrochemistry of the platinum/perfluorosulfonic acid membrane interface.
}

\author{
Alice E. S. Sleightholme and Anthony Kucernak* \\ Department of Chemistry, Imperial College, London SW7 2AZ, UK
}

\begin{abstract}
A solid-state cell is used to study the electrochemistry of platinum at a perfluorosulfonic acid membrane. An anomalous peak is observed in the platinum electrochemistry at approximately $0.6-0.65 \mathrm{~V}$ vs. RHE. The plausible origins of this feature are discussed and experiments which were carried out to characterise the conditions under which the anomalous peak is observed are described. Experiments rule out the possibility of contamination and show that conditions of slow scan rate and low membrane hydration facilitate the appearance of the peak. Scan rate tests indicate that the anomalous feature owes to a surface process. A possible explanation for the origin of the peak is the formation of oxygenated species on the platinum surface.
\end{abstract}

\section{Keywords}

Platinum; Electrocatalyst; perfluorosulfonic acid membrane; Solid-state cell 


\section{Introduction}

The electrochemistry of fuel cell relevant reactions on metal electrodes is usually studied in aqueous electrolytes such as sulfuric acid or perchloric acid. Such electrolytic solutions provide a source of anions that can competitively adsorb on the metal catalyst surface [1], lowering the observed rates of reactions such as methanol oxidation and oxygen reduction. For perfluorosulfonic acid (PFSA) electrolyte membranes, in which the anionic groups have reduced mobility as they are tethered to a polymer backbone, it might be expected that the degree of anion adsorption is decreased, and as a result platinum electrochemistry and electrode kinetics show differences to aqueous acid electrolytes[2]. It is therefore important to study fuel cell relevant reactions at the platinum/PFSA membrane interface in the absence of a supporting liquid electrolyte. Platinum electrochemistry at PFSA membranes is poorly understood and there is limited literature in this area $[2-4]$.

This paper presents a study of platinum/PFSA interfacial electrochemistry using microelectrodes contacting a PFSA electrolyte, in a solid-state electrochemical cell. In the past this experimental approach has previously been used to examine electrochemical reactions such as hydrogen oxidation [2], methanol oxidation [4] and oxygen reduction [5-9]. An anomalous oxidative peak is observed in the voltammetry at $0.6-0.65 \mathrm{~V}$ vs RHE. Similar peaks have been observed in various systems in the literature [10-16], indicating that this may be a universal feature of platinum electrochemistry which is distinguished only under certain experimental conditions. We discuss the plausible origins of this feature and describe experiments which were carried out to characterise the conditions under which the anomalous peak is observed. It is found that the peak may owe to a hitherto unconsidered surface-diffusional process. 


\section{Experimental}

\subsection{Chemicals}

The catalyst studied was a high surface area unsupported platinum catalyst (HiSPEC ${ }^{\text {TM }}$ 1000, 97\%, Johnson Matthey, $\left.27 \mathrm{~m}^{2} \mathrm{~g}_{\mathrm{Pt}}{ }^{-1}\right)$. The electrolytic media used were $0.5 \mathrm{~mol} \mathrm{dm}$ aqueous sulfuric acid (Aristar grade) solution, $0.5 \mathrm{~mol} \mathrm{dm}^{-3}$ aqueous perchloric acid (Aristar grade) solution, Nafion 117 and a hydrocarbon based sulfonic acid cationic-exchange membrane[17] (labelled ' 3542 ' in this paper). The Nafion membranes were cleaned prior to experiments using a standard cleaning procedure consisting of boiling in $3 \%$ hydrogen peroxide, followed by boiling in $0.5 \mathrm{~mol} \mathrm{dm}$ sulfuric acid. Membranes were then rinsed with deionised water $(18 \mathrm{M} \Omega \mathrm{cm}$, Millipore Elix $5 \mathrm{UV}$ water purification system with $<10 \mathrm{ppb}$ total organic content) and immersed in deionised water for storage until experiments were run. The cationic 3542 membrane was hydrated in deionised water overnight. Liquid electrolytic solutions were deoxygenated with research-grade $(99.9999 \%)$ argon prior to the experiments.

\subsection{Preparation of the electrodes}

The working electrodes in all experiments were $60 \mu \mathrm{m}$ gold-wire-in-glass microelectrodes (99.996\% Au, Goodfellow metal). Each microelectrode was polished sequentially with $30 \mu \mathrm{m}, 9$ $\mu \mathrm{m}$, and $0.5 \mu \mathrm{m}$ alumina paper (Agar Scientific). The platinum catalyst was then abrasively attached to a microelectrode using the following method $[18,19]$. A small amount of high-surfacearea metal catalyst was placed on a freshly cleaned glass microscope slide. Then, a microelectrode was rubbed into the catalyst powder to deposit a catalyst layer on the end of the electrode; the presence of deposited catalyst could be observed as a colour change of the microdisk. No ionomer (e.g. Nafion solution) or other electrolyte was added to the microelectrode. From the voltammetric response in sulfuric acid (Fig. 1) and the known surface area of the catalyst we estimate that the electrodes have platinum loading of $2.2 \mathrm{mg} \mathrm{cm}^{-2}$ and a roughness factor of 590 . We estimate that 
the film thickness is approximately $2 \mu \mathrm{m}$ based on the density of platinum and a presumed porosity of 0.5 .

\subsection{Electrochemical measurements}

Voltammetric measurements were performed using an Autolab general-purpose electrochemical system (Ecochemie, Netherlands) using GPES software. All voltammetric scans were started at the lower scan limit unless otherwise stated. A current integrator (Autolab FI20 Filter integrator module) was used to overcome problems associated with the staircase voltammetry of very fast surface processes[20].

In all experiments, the counter electrode was a platinum disk and the reference electrode was a dynamic hydrogen electrode (DHE). Stability of the DHE was monitored by observing the position of the hydrogen adsorption/desorption peaks in the platinum cyclic voltammograms. For the experiments in liquid electrolyte, a three-compartment cell was used with a Luggin capillary for the reference arm and a glass frit between the counter electrode compartment and the main cell. For the experiments using membranes, a solid-state cell was used, as described by Jiang et al. [2, 4]. Unless otherwise stated, in all of the experiments discussed here, $18 \mathrm{M} \Omega \mathrm{cm}$ deionised water was pumped through the cell at $1.2 \mathrm{~cm}^{3} \mathrm{~min}^{-1}$ using a Minipuls 3 peristaltic pump (Gilson, USA) to ensure full membrane hydration. Cartridge heaters mounted within the body of the solid-state cell connected to a PID temperature controller were used to control the cell temperature.

\section{Results}

Figure 1 shows a voltammogram of a high surface area platinum-modified microelectrode cycled at a Nafion 117 membrane. In this voltammogram, the electrode has been pushed into contact with the polymer electrolyte and no ionomer is present in the film. For comparison, a cyclic voltammogram of the same catalyst cycled in $0.5 \mathrm{~mol} \mathrm{dm}^{-3}$ sulfuric acid taken after the above voltammogram (to avoid potential contamination of the electrode with acid) is overlayed. The figure indicates that the electrochemistry of platinum at a Nafion membrane is very different from 
that in liquid acid. The hydrogen adsorption and desorption peaks are much broader in the Nafion $\mathrm{CV}$, and oxide formation and reduction is shifted to lower potentials. These results are expected when moving from an electrolyte containing a strongly absorbing anion $\left(\mathrm{HSO}_{4}{ }^{-}\right)$to the solid polymer electrolyte. The hydrogen absorption peaks provide a charge of about $60 \%$ of that obtained in sulfuric acid, suggesting that the majority of the platinum is involved in the electrochemical reaction. Another significant difference between the two CVs is the extra peak exhibited by the platinum/Nafion system at approximately $0.6-0.65 \mathrm{~V}$ vs RHE. In order to characterise this peak several possible causes were studied.

\subsection{Contamination}

As mentioned above, examples of similar peaks have been reported indicating that this peak might involve aspects of the electrochemistry of platinum particular to those systems. To determine whether such queries were relevant, it was first necessary to eliminate the possibility that the peak arose from the presence of a contaminant. To minimise any contamination in the cell the materials comprising the cell were selected such that they were free of plasticisers, which could potentially leach into the cell. The Nafion membrane was also compared to a different cationic-exchange membrane, 3542, under the same experimental conditions and the peak was observed in both cases. Both the synthetic routes and the structures of the two membranes were very different, and therefore contamination by residual short-chain organic compounds or a remnant synthetic catalyst in the Nafion was considered highly unlikely.

The working electrode was another possible cause of contamination. The solid-state cell experiment was repeated with a liquid electrolyte to test whether any components of the working electrode (the gold electrode, the Nafion binder or the catalyst) were contaminated. A platinummodified gold electrode was cycled first in $0.5 \mathrm{~mol} \mathrm{dm}^{-3}$ perchloric acid, followed by washing with $18 \mathrm{M} \Omega \mathrm{cm}$ deionised water. Next, the same electrode was cycled in $0.5 \mathrm{~mol} \mathrm{dm}{ }^{-3}$ sulfuric acid and washed again with $18 \mathrm{M} \Omega \mathrm{cm}$ deionised water followed by cycling in the solid-state cell with a Nafion membrane. The experiment was carried out identically in all cases with the only difference 
being the electrolyte employed. Figure 2 shows a comparison of cyclic voltammograms for the platinum-modified gold electrode in each electrolyte. As the electrolyte is modified from one containing a strongly adsorbing anion $\left(\mathrm{HSO}_{4}^{-}\right)$to one containing a weakly adsorbing anion $\left(\mathrm{ClO}_{4}^{-}\right)$, there is a broadening of both the hydrogen adsorption and oxide formation peaks. This trend continues when the electrolyte is changed to Nafion. The anomalous peak is not apparent in the voltammograms measured in liquid electrolytic solutions. However, the peak appears when the same electrode is removed from the acid and tested in contact with the Nafion membrane electrolyte. This suggests that the peak does not owe to a contaminant present on the electrode because it reappears even after the electrode has been cycled in acid and washed with water.

Both the counter electrode and catalyst itself might also have supplied contaminants. The type of counter electrode was determined to be uncorrelated to the appearance of the peak. A high surface area palladium catalyst was tested under the same conditions as the platinum (results not shown) and in this case no anomalous peak was observed. Palladium and platinum are similar enough to suggest that if the peak were due to a contaminant, then it would also be seen with the palladium catalyst. This suggests that the reaction producing the peak arises due to a process specific to platinum catalysts. This also indicates that the peak does not owe to contamination, as the peak would also appear in the palladium voltammetry if this were the case. An alternative, high surface area mesoporous platinum catalyst [21] was also studied in the solid-state cell under the same experimental conditions. In this case the anomalous peak was observed at the same potential. Again, this indicates that the peak is not a contamination effect.

\subsection{Water flow through the solid-state cell}

The effect of membrane hydration was tested by altering the water flow through the solidstate cell. When water was pumped through the cell, the peak was found to decrease in size. Initially, this was thought to indicate that a contaminant was present which was convected away with water. However, with no water flow, the peak remained the same size, even after 20 hours of repeated cycling. This suggested that if a contaminant was the cause of the peak, it was not in 
limited supply. The anomalous peak was also shown to disappear with water flow and then reappear gradually as the cell was left to rest with no water flow. Figure 3 shows cyclic voltammograms for a platinum-modified $60 \mu \mathrm{m}$ microelectrode cycled at a Nafion 117 membrane at room temperature with water pumped through the cell for 5 hours, followed by 65 hours rest, and then pumped for a final 5 hours. The scans at 70 and 75 hours have been shifted on the voltage axis to account for the reference electrode shift which occurred over the long time periods. The anomalous peak was shown to disappear with water flow and then reappear as the cell rested. Such behaviour further contradicts the hypothesis that water flow extracts a contaminant from the system, unless the contaminant is present in very large supply. This was very unlikely as all parts of the set up were individually tested, and no evidence of contamination was found.

To ensure that water flow had no effect on the size of the peak when other variables were being studied, no water was pumped through the cell during the remaining experiments reported in this paper.

\subsection{Environmental Effects}

The effects of oxygen, hydrogen and hydrogen peroxide (the standard potential of oxidation of $\mathrm{H}_{2} \mathrm{O}_{2}$ is $0.68 \mathrm{~V}$ vs RHE [22]) on the occurrence of the anomalous peak were studied.

For the tests involving oxygen and hydrogen, the experiments were carried out with no water flow through the cell and, instead, each of the gases was pumped through the cell for several hours with cyclic voltammograms measured continually at $5 \mathrm{mV} \mathrm{s}^{-1}$. In both cases, there was no change in the size of the anomalous peak over the experimental time period, indicating that the presence of molecular hydrogen and oxygen did not have a significant effect on the peak in the platinum scan.

For tests involving hydrogen peroxide, the cell was run with flowing water. This allowed the addition of varying amounts of hydrogen peroxide to the water as the electrode was scanned continuously at $5 \mathrm{mV} \mathrm{s}^{-1}$. The anomalous peak was shown to be present initially and was suppressed as water was pumped into the cell, identical to the experiments without peroxide. There was no 
increase in the size of the peak as peroxide concentration was increased, indicating that the cause of

the peak was not the presence of hydrogen peroxide.

\subsection{The effect of temperature}

Figure 4 shows cyclic voltammograms for a platinum-modified $60 \mu \mathrm{m}$ electrode cycled at a Nafion 117 membrane at various temperatures. The cell was first cycled at $60{ }^{\circ} \mathrm{C}$, the temperature was then lowered to $40{ }^{\circ} \mathrm{C}$, and finally to $28{ }^{\circ} \mathrm{C}$. The height of the anomalous oxidation peak is shown to be greater at higher temperatures, indicating that an activated reaction is occurring. It also shows a shift to more negative potentials at higher temperatures. Although the hydrogen adsorption region gave a consistent area as a function of temperature, the oxide formation and reduction regions increased with size at higher temperature.

\subsection{The effect of change in potential window}

The potential window used in the cyclic voltammetry described so far was $0 \mathrm{~V}$ to $1.4 \mathrm{~V}$ vs RHE. In order to characterise the conditions under which the anomalous peak appears more precisely, the potential window was adjusted by modifying the lower scan limit potential in $0.04 \mathrm{~V}$ steps from $0.0 \mathrm{~V}$ to $0.4 \mathrm{~V}$ vs RHE. Figure 5 shows cyclic voltammograms for a platinum-modified $60 \mu \mathrm{m}$ microelectrode cycled against a Nafion 117 membrane, at room temperature. The figure shows that the anomalous peak shrinks as the lower bound of the voltage range increases from $0 \mathrm{~V}$ to $0.4 \mathrm{~V}$. The peak disappears when the lower limit is more positive than approximately $0.2 \mathrm{~V}$ vs RHE.

This observation was shown to be reproducible by alternating the potential window used. The results show that when the lower limit of the potential window is varied between potentials more negative of $0.2 \mathrm{~V}$ vs RHE and potentials more positive of $0.2 \mathrm{~V}$ vs RHE, the size of the anomalous peak reversibly increases and decreases, respectively.

The results suggest that the effect of changing the potential window may arise from cycling of the electrode to potentials within the hydrogen adsorption/desorption region of platinum; however it may also owe to the amount of time allowed for whatever reaction produces the peak 
(i.e. as the lower scan limit is increased, the potential associated with the anomalous peak is reached at an earlier time). To test the later hypothesis, the starting potential was held for a predefined period at the lower scan limit before the scan commenced. Figure 6 shows the resulting cyclic voltammograms for a platinum-modified $60 \mu \mathrm{m}$ electrode cycled at a Nafion 117 membrane. An initial equilibration step was carried out where the electrode was held at $0.40 \mathrm{~V}$ vs RHE for 1 second to ensure that the initial state of the electrode was the same in all cases (i.e., free of adsorbed hydrogen). The potential was then held for either 10 (figure 6a) or 100 (figure 6b) seconds at the lower potential limit before voltammetry commenced. The longest hold time of 100 seconds was chosen as it is greater than the 80 -second time period taken to cycle between 0 and $0.4 \mathrm{~V}$ at $5 \mathrm{mV} \mathrm{s}^{-1}$. The voltammograms show that when the potential is held for 10 seconds, the peak appears to reduce gradually as the potential at which the electrode is held becomes more positive. This is similar to the voltammograms shown in figure 5. With a hold time of 100 seconds, however, the peak is significantly larger at every holding potential, even those for which it was barely visible at shorter hold times. When the potential is held at $0 \mathrm{~V}$ vs RHE there is little change in peak size as hold time increases from 10 seconds to 100 seconds. However, when the baseline potential is $0.4 \mathrm{~V}$ vs RHE, the peak height increases dramatically with increasing hold time. This suggests that time is an important factor in determining the size of the extra oxidation peak and that the reaction which occurs to produce the peak is slow.

\subsection{The effect of scan rate}

At fast scan rates (above approximately $13 \mathrm{mV} \mathrm{s}^{-1}$ ) no peak at $0.6-0.65 \mathrm{~V}$ vs RHE is visible in the cyclic voltammograms. Clearly scan rate has a significant impact on the peak size. As the time elapsed when cycling the platinum catalyst between the starting potential and the anomalous peak potential appears to be an important factor in determining the peak height, it was important to use an appropriate potential window when varying the scan rate. Before each scan rate the potential was held at $0.4 \mathrm{~V}$ vs RHE for 200 seconds before cycling between $0.4 \mathrm{~V}$ and $1.0 \mathrm{~V}$ vs RHE. The electrode was scanned from $1 \mathrm{mV} \mathrm{s}^{-1}$ to $20 \mathrm{mV} \mathrm{s}^{-1}$. Figure 7 shows a plot of peak current 
as a function of scan rate for a platinum-modified $60 \mathrm{~mm}$ gold electrode cycled at a Nafion 117 membrane. The current associated with the anomalous peak in the platinum scan was calculated by taking the height of the peak relative to a linearly interpolated baseline. The plot produces a linear graph. In comparison, a plot of peak current as a function of square root scan rate is fit well by a parabola. This result suggests that the anomalous peak probably owes to a surface process.

\section{Discussion}

From the results described in this paper it can be concluded that the anomalous oxidation peak observed at the platinum/PFSA membrane interface only appears under a narrow range of experimental conditions. Comparative results obtained in liquid electrolytic solutions have shown that the feature only appears when platinum is electrochemically cycled in contact with a PFSA membrane. Under otherwise identical conditions in liquid electrolyte, no anomalous peak is observed. Water flow through the cell eliminates the peak, so characterisation of the peak is only possible when the membrane is hydrated by immobile water. The anomalous peak merges into the background when the platinum/PFSA interface is electrochemically cycled above a scan rate of 13 $\mathrm{mV} \mathrm{s}^{-1}$. A change in the potential window can also eliminate the peak when the lower potential window limit extends above $0.2 \mathrm{~V}$ vs RHE. However, the peak can reappear with such potential windows after prepolarisation of at least 100 seconds.

As mentioned earlier, some similar peaks have been observed elsewhere in the literature, in systems with platinum $(111)[11,15,16]$ and platinum systems cycled in highly concentrated liquid-acid electrolytes $[10,12-14]$. The literature provides a number of hypothetical electrochemical mechanisms that may lead to the peak, which are considered below.

\subsection{Platinum electrochemistry in highly concentrated acid electrolyte}

Vogel and Baris [10] provide cyclic voltammograms for a platinum wire cycled in $90 \%$ phosphoric acid at $160{ }^{\circ} \mathrm{C}$. In this experiment, an unusual oxidation peak was observed at approximately $0.7 \mathrm{~V}$ vs RHE and was shown to grow with the duration of prepolarisation at $0.1 \mathrm{~V}$ vs RHE. A similar peak is observed, in this case for platinum foil cycled in $86 \%$ phosphoric acid, 
by Clouser and co-workers [12]. Again, this peak was observed at approximately $0.7 \mathrm{~V}$ vs RHE and

appeared only above $60{ }^{\circ} \mathrm{C}$. In this experiment the potential window was also investigated; the peak was only present when the lower potential limit was below $0.4 \mathrm{~V}$ vs RHE. Clouser et al.'s result confirms the results described in section 3.5 , and illustrated in figure 5 .

Vogel et al. and Clouser et al. both attribute the peak to the oxidation of reduction byproducts of phosphoric acid. However, in a later study by Burke and co-workers, [13] which showed similar results, it was suggested that this conclusion was incorrect. Burke et al. studied polycrystalline platinum in $1 \mathrm{~mol} \mathrm{dm}^{-3}$ phosphoric acid at varying temperatures. Two unusual peaks were observed at $0.5 \mathrm{~V}$ and $0.7 \mathrm{~V}$ vs RHE, potentials similar to those of which the peak was observed here. Burke et al. used much lower phosphoric acid concentration and lower temperatures than either Clouser et al. or Vogel and Baris. The presence of impurities was ruled out, and it was concluded that the peaks arose from 'premonolayer oxidation processes'. This hypothesis is supported by the observation of a similar peak at approximately $0.65 \mathrm{~V}$ vs RHE in a system involving sulfuric acid at $80{ }^{\circ} \mathrm{C}$ [14]. Its presence in this case contradicts the hypothesis that the peak is due to the decomposition of phosphoric acid. The anomaly appears to be similar in different acids and it has been suggested that it is a property of the metal rather than of the electrolyte [13].

The present results for platinum/PFSA interfaces are similar enough to those for platinum in highly concentrated acid solutions that it seems plausible the anomalous peaks have the same origin. In all cases the peaks occur at similar potentials, appear in low water environments, and grow significantly with increasing temperature. Taken together the observations argue strongly that the anomalous peak may be a feature of platinum electrochemistry in low water environments.

\subsection{Formation of $\mathrm{OH}$ at platinum}

Adsorbed oxygencontaing compounds labelled as "OH" typically forms on polycrystalline platinum in the range of $0.85-0.90 \mathrm{~V}$ vs RHE. This peak shifts to lower potentials when $\mathrm{OH}$ is formed on more structured surfaces, for instance on a uniform platinum (111) surface. In contrast, the formation of $\mathrm{OH}$ shifts to higher potentials when more strongly adsorbing anions are present in 
the electrolytic solution. Since Nafion (or other PFSA membrane) provides no free adsorbing

anions, the formation of oxide may be expected to shift to lower potentials than those expected for platinum in liquid acids. Anion adsorption may still occur, but its extent is probably severely limited since sulphonic acid groups are bound to Nafion's perfluorinated backbone. Formation of $\mathrm{OH}$ as the platinum catalyst is electrochemically cycled in contact with a PFSA membrane may be a plausible explanation for the origin of the anomalous peak.

\subsubsection{Single-crystal platinum (111)}

Many studies have been carried out on the electrochemistry of single-crystal platinum [15, 16, 23-37]. The two most typical techniques used to prepare platinum single-crystal electrodes are ultrahigh vacuum preparation [37] or flame annealing [28]. Both techniques yield platinum (111) surfaces which show 'butterfly' peaks in acid at approximately $0.5-0.7 \mathrm{~V}$ vs RHE. As both preparation techniques produce similar results, this suggests that the 'butterfly' peaks do not owe to impurities electrode surface or in the electrolyte [16]. It has also been shown that the peaks appear regardless of the electrolyte anion; they have been observed for Pt (111) cycled in both sulfuric acid $[16,28,29]$ and perchloric acid $[15,16]$. It is believed that these peaks signify anion adsorption (i.e., the adsorption of $\mathrm{HSO}_{4}^{-}$in the case of sulfuric acid and the adsorption of $\mathrm{OH}^{-}$in the case of perchloric acid $[16,35,37])$. The 'butterfly' peaks occur at approximately the same potential as the anomalous peak reported in this paper. Again, the peaks may have similar origins.

High surface area platinum may contain a high surface ratio of platinum (111) because that is the most thermodynamically stable crystal surface. It is, therefore, possible that it is the electrochemistry of the Pt (111) orientations that produces the anomalous peak observed when a high surface area platinum catalyst is electrochemically cycled at a PFSA membrane. However, results from the experiments where a platinum electrode was cycled in liquid electrolytic solutions contradict this explanation as the anomalous peak was only observed when studied with a PFSA membrane. The anomalous peak at the platinum/Nafion interface was also shown to appear only at slow scan rates which, again, contradicts this theory as the research on platinum (111) in acid shows 
the 'butterfly' peaks at much faster scan rates. From these comparisons, it does not seem likely that the anomalous peak described in this paper owes to the formation of 'butterfly peaks' due the catalyst containing a high surface ratio of (111) crystallographic orientations. Although the conditions where platinum (111) exhibits the peak differ from those explored here, the origin of the peak may still be mechanistically similar in both cases, arising from $\mathrm{OH}$ formation on the platinum surface.

\subsubsection{Low water environment}

The effect of water flow through the electrochemical cell on the size and presence of the anomalous peak was described in section 3.2. It was shown that as water was pumped into the cell it had the effect of decreasing the size of the peak. It was also shown that this was reversible - when the cell was left to rest with no water flow, the peak reappeared.

The effect of water flow on the experimental results may owe to changes in hydration of the Nafion (or other PFSA) membrane. Recently it has been proposed that as Nafion hydrates, the morphology changes from a cluster-like structure to a rod-like structure [38]. As the membrane swells, the ionic domains increase in size and channels form. At this level of hydration, $\mathrm{H}^{+}$ions can move through the channels in the membrane. Above $50 \%$ water volume fraction there is a structure inversion and the sulphonic acid groups switch from being on the inside of the channels to the outside of the polymer domains. After further hydration, the structure changes from cluster-like to a connected network of rods.

The inversion of the membrane structure as it swells may impact the electrochemistry of the platinum catalyst. In a more hydrated state, the polymer domains become more mobile and sulphonic acid groups may have greater access to the catalyst surface. This would explain the decrease in the relative size of the anomalous peak as water is pumped into the cell, as the adsorption of anions onto the surface, allowed by greater hydration, would compete with the reaction occurring to produce the peak.

It therefore seems plausible that the anomalous peak observed in the electrochemistry of the 
platinum/PFSA interface may owe to formation of oxygenated species. This formation is apparently slow, and may be suppressed under conditions of high water content, in which Nafion inverts its structure anion adsorption competes with the reaction which produces the peak.

\section{Conclusion}

The electrochemistry of the platinum/PFSA membrane interface has been shown to differ significantly from that of platinum/liquid-acid interfaces. The most significant difference is the addition of an anomalous oxidation peak in the electrochemical voltammetry at approximately $0.6-$ $0.65 \mathrm{~V}$ vs RHE. The peak has been shown to feature in the electrochemistry of platinum catalysts only; in comparison with a palladium catalyst, no peak appeared. Experiments have ruled out the possibility of contamination and have shown that conditions of slow scan rate and low membrane hydration facilitate the appearance of the peak. It increases in size and shifts to lower potentials with an increase in temperature; scan rate tests indicate that it is due to a surface process. The peak is observed only when the catalyst is cycled negative of $0.2 \mathrm{~V}$ vs RHE or when the electrode is prepolarised, indicating that the reaction which occurs to produce the peak is slow.

Similar peaks were reported in studies of the electrochemistry of polycrystalline platinum in highly concentrated acids, and also in the electrochemistry of platinum (111). The structure of hydrated Nafion was considered as a possible explanation of the effect of water on the anomalous peak. As it seems conclusive that the peak does not owe to a contaminant, a possible explanation for the origin of the peak would be the formation of oxygenated species on the platinum surface. The disappearance of the peak when water is pumped into the cell or when the catalyst is cycled in liquid acid electrolytes could then be explained by anion adsorption.

\section{Acknowledgements}

Alice Sleightholme thanks the EPSRC for studentship funding. 
References

1. Sobkowski, J. and A. Wieckowski, The influence of halide ions on methanol adsorption and oxidation on a platinised electrode. Journal of Electroanalytical Chemistry, 1973. 41: p. 373-379.

2. Jiang, J. and A. Kucernak, Investigations of fuel cell reactions at the composite microelectrode/solid polymer electrolyte interface 1. Hydrogen oxidation at the nanostructured Pt/Nafion membrane interface. Journal of electroanalytical Chemistry, 2004. 567: p. 123-137.

3. Paik, W.K., T.E. Springer, and S. Srinivasan, Kinetics of fuel cell reactions at the platinum/solid polymer electrolyte interface. Journal of the Electrochemical Society, 1989. 136(3): p. 644-9.

4. Jiang, J. and A. Kucernak, Solid polymer electrolyte membrane composite microelectrode investigations of fuel cell reactions. II: voltammetric study of methanol oxidation at the nanostructured platinum microelectrode|Nafion membrane interface. Journal of Electroanalytical Chemistry, 2005. 576(2): p. 223-236.

5. Parthasarathy, A., C.R. Martin, and S. Srinivasan, Investigations of the molecular oxygen reduction reaction at the platinum/Nafion interface using a solid-state electrochemical cell. Journal of the Electrochemical Society, 1991. 138(4): p. 916-21.

6. Parthasarathy, A., S. Srinivasan, and A.J. Appleby, Temperature dependence of the electrode kinetics of oxygen reduction at the platinum/Nafion interface. A microelectrode investigation. Journal of the Electrochemical Society, 1992. 139(9): p. 2530-7.

7. Basura, V.I., P.D. Beattie, and S. Holdcroft, Solid-state electrochemical oxygen reduction at Pt/Nafion 117 and Pt/BAM3G 407 interfaces. Journal of Electroanalytical Chemistry, 1998. 458(1-2): p. 1-5.

8. Beattie, P.D., V.I. Basura, and S. Holdcroft, Temperature and pressure dependence of O2 reduction at Pt. Nafion 117 and Pt. BAM 407 interfaces. Journal of Electroanalytical Chemistry, 1999. 468(2): p. 180-192.

9. $\quad$ Sleightholme, A.E.S., J.R. Varcoe, and A.R. Kucernak, Oxygen reduction at the silver/hydroxide-exchange membrane interface. Electrochem. Commun. FIELD Full Journal Title:Electrochemistry Communications, 2008. 10(1): p. 151-155.

10. Vogel, W.M. and J.M. Baris, Changes in the surface of platinum in hot concentrated phosphoric acid at low potentials. Electrochimica Acta, 1978. 23(5): p. 463-6.

11. Iwasita, T. and X. Xia, Adsorption of water at Pt(111) electrode in HClO4 solutions. The potential of zero charge. Journal of Electroanalytical Chemistry, 1996. 411(1-2): p. 95-102.

12. Clouser, S.J., J.C. Huang, and E. Yeager, Temperature dependence of the Tafel slope for oxygen reduction on platinum in concentrated phosphoric acid. Journal of Applied Electrochemistry, 1993. 23(6): p. 597-605.

13. Burke, L.D. and J.A. Morrissey, Hydrous oxide formation on platinum in phosphoric acid solution. Journal of the Electrochemical Society, 1994. 141(9): p. 2361-8.

14. Burke, L.D. and J.K. Casey, The role of hydrous oxide species on platinum electrocatalysts in the methanol/air fuel cell. Electrochimica Acta, 1992. 37(10): p. 1817-29.

15. Markovic, N.M., et al., Structural effects in electrocatalysis: oxygen reduction on platinum low index single-crystal surfaces in perchloric acid solutions. Journal of Electroanalytical Chemistry, 1994. 377(1-2): p. 249-59.

16. Al Jaaf-Golze, K., D.M. Kolb, and D. Scherson, The voltammetry curves of platinum(111) in aqueous solutions. Journal of Electroanalytical Chemistry and Interfacial Electrochemistry, 1986. 200(1-2): p. 353-62.

17. Horsfall, J.A. and K.V. Lovell, Synthesis and characterization of sulfonic acid-containing ion exchange membranes based on hydrocarbon and fluorocarbon polymers. European Polymer Journal 2002. 38(8): p. 1671-1682. 
18. Jiang, J.J. and A. Kucernak, Oxygen reduction studies of templated mesoporous platinum catalysts. Electrochemical and Solid State Letters, 2000. 3(12): p. 559-562.

19. Cha, C.S., et al., Powder microelectrodes. Journal of Electroanalytical Chemistry, 1994. 368(1-2): p. 47-54.

20. Comparison between staircase cyclic voltammetry and cyclic voltammetry, linear scan. AUTOLAB APPLICATION NOTE [cited; Available from: http://www.ecochemie.nl/download/content/Appl021.pdf.

21. Jiang, J.H. and A. Kucernak, Electrooxidation of small organic molecules on mesoporous on precious metal catalysts $I$ : CO and methanol on platinum. Journal of Electroanalytical Chemistry, 2002. 533(1-2): p. 153-165.

22. CRC Handbook of Chemistry and Physics. 52nd Edition, 1971-1972.

23. Clavilier, J., The role of anion on the electrochemical behavior of a $\{111\}$ platinum surface; an unusual splitting of the voltammogram in the hydrogen region. Journal of Electroanalytical Chemistry and Interfacial Electrochemistry, 1980. 107(1): p. 211-16.

24. Kita, H., et al., Adsorption of hydrogen on platinum single crystal electrodes in acid and alkali solutions. Journal of Electroanalytical Chemistry and Interfacial Electrochemistry, 1990. 295(1-2): p. 317-31.

25. Yeager, E., et al., Hydrogen adsorption on single crystal platinum. Journal of the Electrochemical Society, 1978. 125(2): p. 348-9.

26. Hubbard, A.T., R.M. Ishikawa, and J. Katekaru, Study of platinum electrodes by means of electrochemistry and low-energy electron diffraction. Part II. Comparison of the electrochemical activity of Pt(100) and Pt(111) surfaces. Journal of Electroanalytical Chemistry and Interfacial Electrochemistry, 1978. 86(2): p. 271-88.

27. Yamamoto, K., et al., Hydrogen adsorption and oxide formation on platinum single crystal electrodes. Journal of Electroanalytical Chemistry and Interfacial Electrochemistry, 1978. 96(2): p. 233-9.

28. Clavilier, J., et al., Preparation of monocrystalline platinum microelectrodes and electrochemical study of the plane surfaces cut in the direction of the $\{111\}$ and $\{110\}$ planes. Journal of Electroanalytical Chemistry and Interfacial Electrochemistry, 1980. 107(1): p. 205-9.

29. Funtikov, A.M., U. Stimming, and R. Vogel, Anion adsorption from sulfuric acid solutions on Pt(111) single crystal electrodes. Journal of Electroanalytical Chemistry, 1997. 428(1-2): p. 147-153.

30. Clavilier, J., D. Armand, and B.L. Wu, Electrochemical study of the initial surface condition of platinum surfaces with (100) and (111) orientations. Journal of Electroanalytical Chemistry and Interfacial Electrochemistry, 1982. 135(1): p. 159-66.

31. Kita, H., H.-W. Lei, and Y. Gao, Oxygen reduction on platinum single-crystal electrodes in acidic solutions. Journal of Electroanalytical Chemistry, 1994. 379(1-2): p. 407-14.

32. Markovic, N., et al., The effects of anions on hydrogen electrosorption on platinum singlecrystal electrodes. Journal of Electroanalytical Chemistry and Interfacial Electrochemistry, 1986. 214(1-2): p. 555-66.

33. Markovic, N.M., B.N. Grgur, and P.N. Ross, Temperature-dependent hydrogen electrochemistry on platinum low-index single-crystal surfaces in acid solutions. Journal of Physical Chemistry B, 1997. 101(27): p. 5405-5413.

34. Markovic, N.M., et al., Hydrogen electrochemistry on platinum low-index single-crystal surfaces in alkaline solution. Journal of the Chemical Society, Faraday Transactions, 1996. 92(20): p. 3719-3725.

35. Marinkovic, N.S., N.M. Markovic, and R.R. Adzic, Hydrogen adsorption on single-crystal platinum electrodes in alkaline solutions. Journal of Electroanalytical Chemistry, 1992. 330(1-2): p. 433-52.

36. Motoo, S. and N. Furuya, Electrochemistry of platinum single crystal surfaces. Part I. Structural change of the platinum(111) surface followed by an electrochemical method. 
Journal of Electroanalytical Chemistry and Interfacial Electrochemistry, 1984. 172(1-2): p. 339-58.

37. Wagner, F.T. and P.N. Ross, Jr., LEED analysis of electrode surfaces. Structural effects of potentiodynamic cycling on platinum single crystals. Journal of Electroanalytical Chemistry and Interfacial Electrochemistry, 1983. 150(1-2): p. 141-64.

38. Gebel, G., Structural evolution of water-swollen perfluorosulfonated ionomers from dry membrane to solution. Polymer, 2000. 41(15): p. 5829-5838. 


\section{Captions}

Figure $1 \mathrm{~A}$ comparison of platinum electrochemistry at $0.5 \mathrm{~mol} \mathrm{dm}^{-3} \mathrm{H}_{2} \mathrm{SO}_{4}(---)$ and Nafion 117 (-) electrolytes. Cyclic voltammograms measured for a high surface area platinum modified 60 $\mu \mathrm{m}$ gold electrode, at a scan rate of $5 \mathrm{mV} \mathrm{s}^{-1}$, at room temperature. The anomalous feature is circled.

Figure 2 A comparison of electrolytes: $0.5 \mathrm{~mol} \mathrm{dm}^{-3} \mathrm{H}_{2} \mathrm{SO}_{4}, 0.5 \mathrm{~mol} \mathrm{dm}^{-3} \mathrm{HClO}_{4}$ and Nafion 117 . Cyclic voltammograms measured for a platinum-modified $60 \mu \mathrm{m}$ gold electrode at a scan rate of 5 $\mathrm{mV} \mathrm{s}^{-1}$, at room temperature.

Figure 3 Cyclic voltammograms for a platinum-modified $60 \mu \mathrm{m}$ gold electrode cycled at a Nafion 117 membrane at room temperature. Voltammogram for initial system $(1),(-)$.Water pumped through cell for 5 hours (2), ( …...); 65 hours rest with no water flowing through cell (3), (-); and water flowing through cell for a further 5 hours, (4), (….... Scan rate $5 \mathrm{mV} \mathrm{s}^{-1}$. Solid lines indicate no water flow through cell and dashed lines indicate water flow through cell.

Figure 4 Cyclic voltammograms for a platinum-modified $60 \mu \mathrm{m}$ gold electrode cycled at a Nafion 117 membrane at $28{ }^{\circ} \mathrm{C}(-) 40{ }^{\circ} \mathrm{C}(---)$ and $60{ }^{\circ} \mathrm{C}(\cdots \cdots \cdots)$ with no water flow though the cell. Scan rate $5 \mathrm{mV} \mathrm{s}^{-1}$.

Figure 5 Cyclic voltammograms for a platinum-modified $60 \mu \mathrm{m}$ gold electrode cycled at a Nafion 117 membrane at a scan rate of $5 \mathrm{mV} \mathrm{s}^{-1}$ and at room temperature with no water flow through cell. Arrows indicate the change in peaks as the potential window lower limit was altered from $0 \mathrm{~V}$ to $0.4 \mathrm{~V}$ vs RHE. 
Figure 6 Cyclic voltammograms for a platinum-modified $60 \mu \mathrm{m}$ gold electrode cycled at a Nafion membrane with no water flow through cell at a scan rate of $5 \mathrm{mV} \mathrm{s}^{-1}$ and at room temperature. Potential held for a) 10 seconds and b) 100 seconds at lower limit potentials. Arrows indicate the change in peaks as the potential window lower limit was altered from $0 \mathrm{~V}$ to $0.4 \mathrm{~V}$ vs RHE.

Figure 7 Plot of peak current as a function of scan rate for a platinum-modified $60 \mu \mathrm{m}$ gold electrode cycled at a Nafion membrane. Electrode prepolarised at $0.4 \mathrm{~V}$ vs. RHE for 200s followed by cycling from that potential to $1.0 \mathrm{~V}$ vs. RHE at a scan rate of $1-20 \mathrm{mVs}^{-1}$. The peak current was deconvoluted from the rising background by using a linear background interpolation. 
Figures 1-7

Figure 1

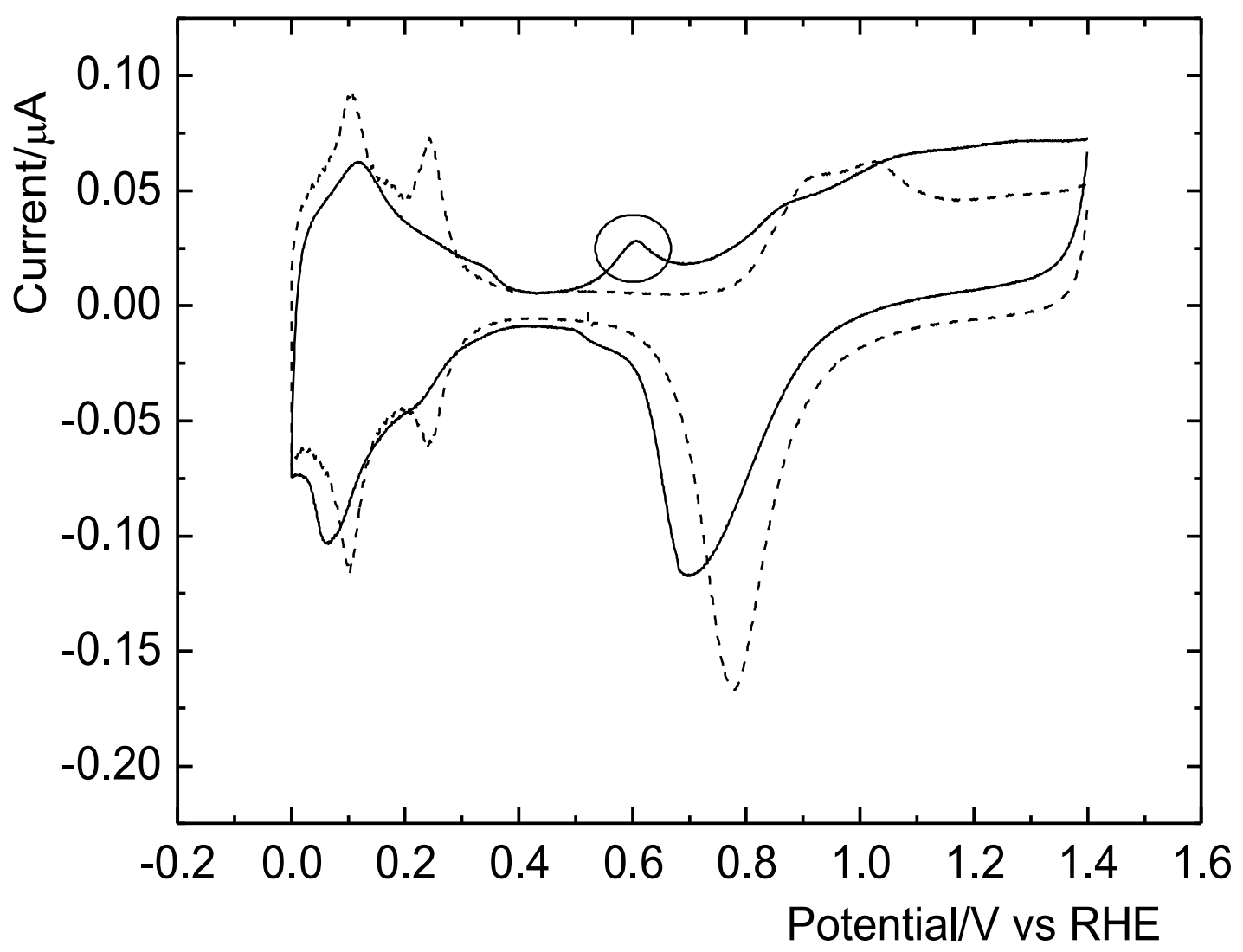


Figure 2

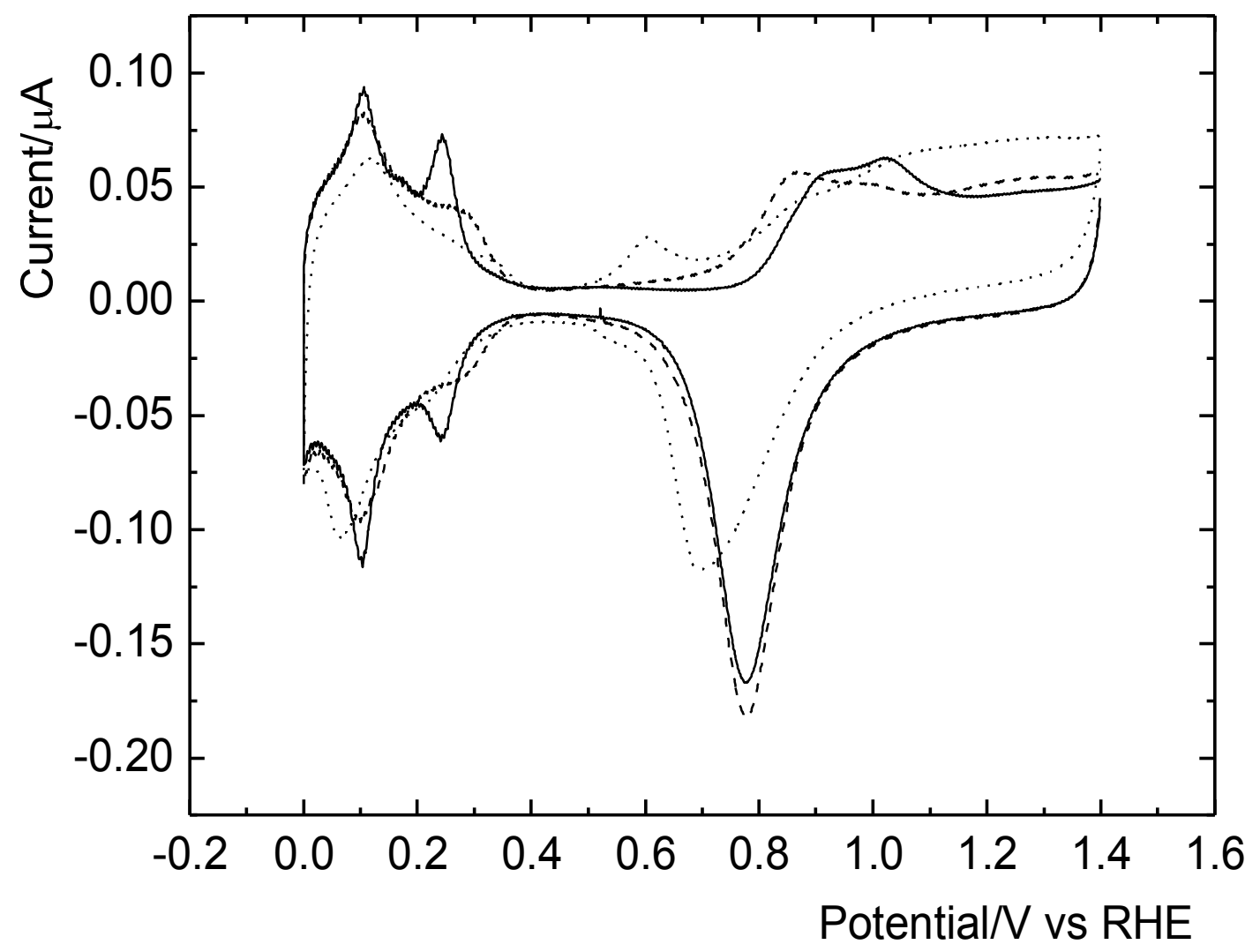


Figure 3




Figure 4

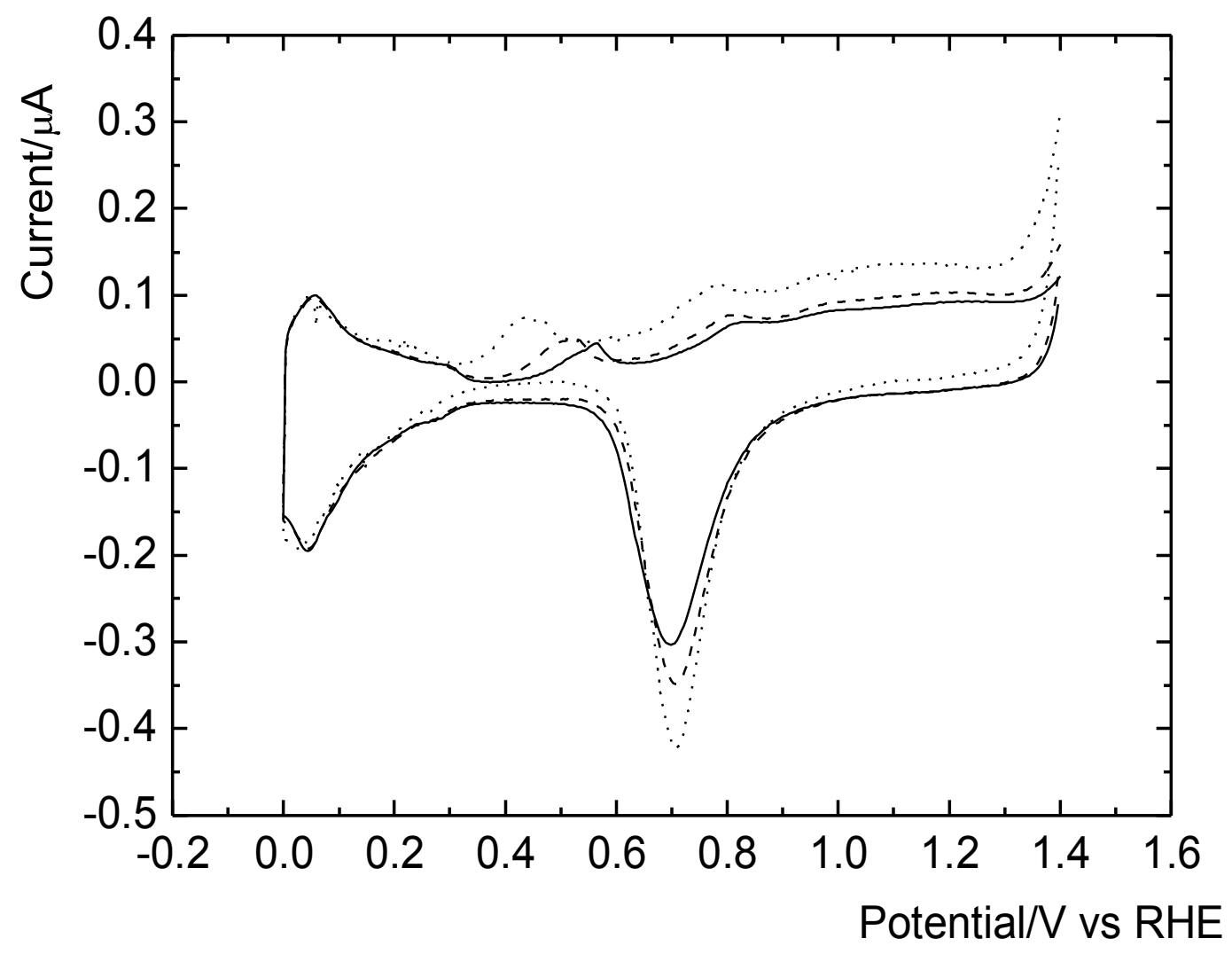


Figure 5

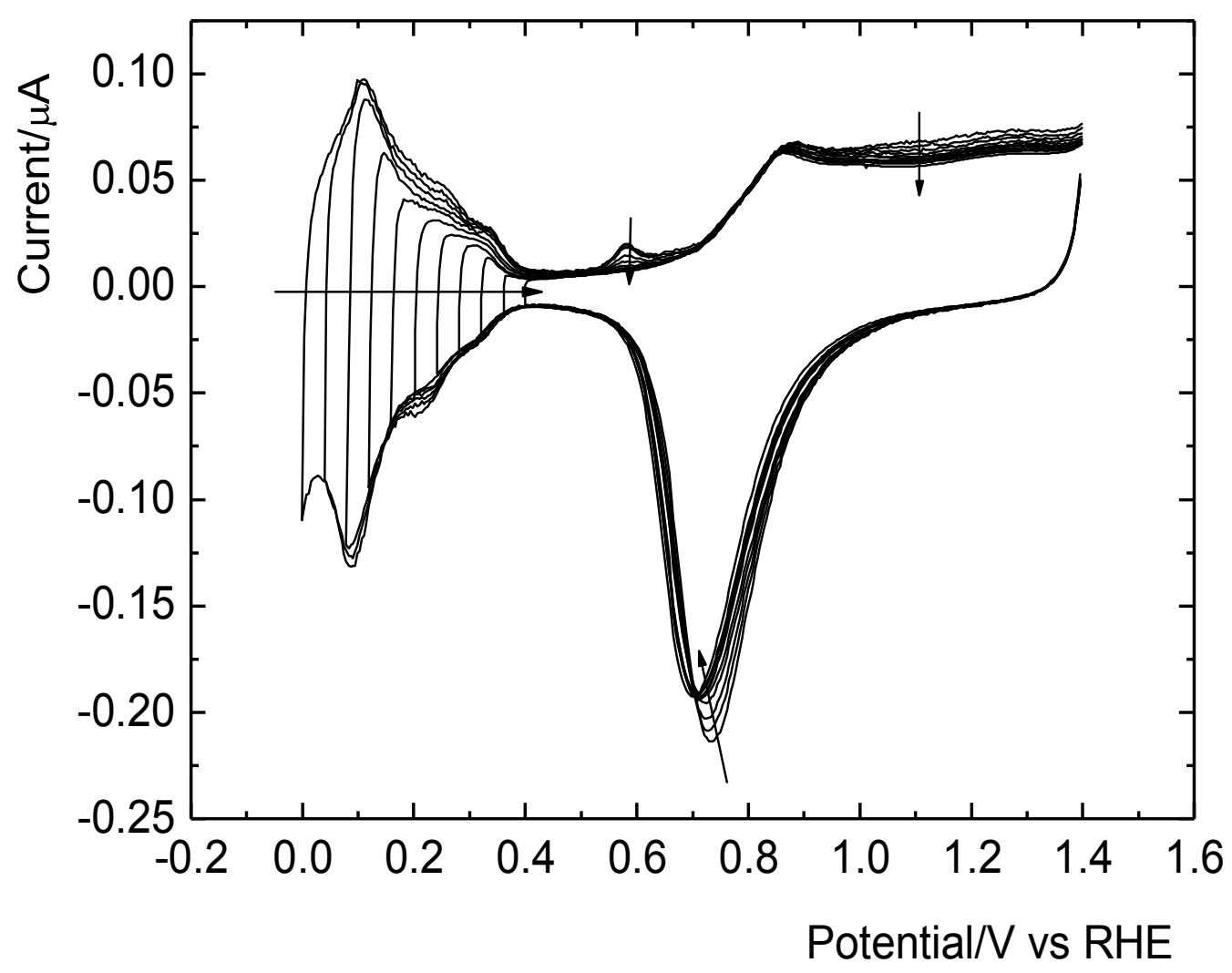


Figure 6a

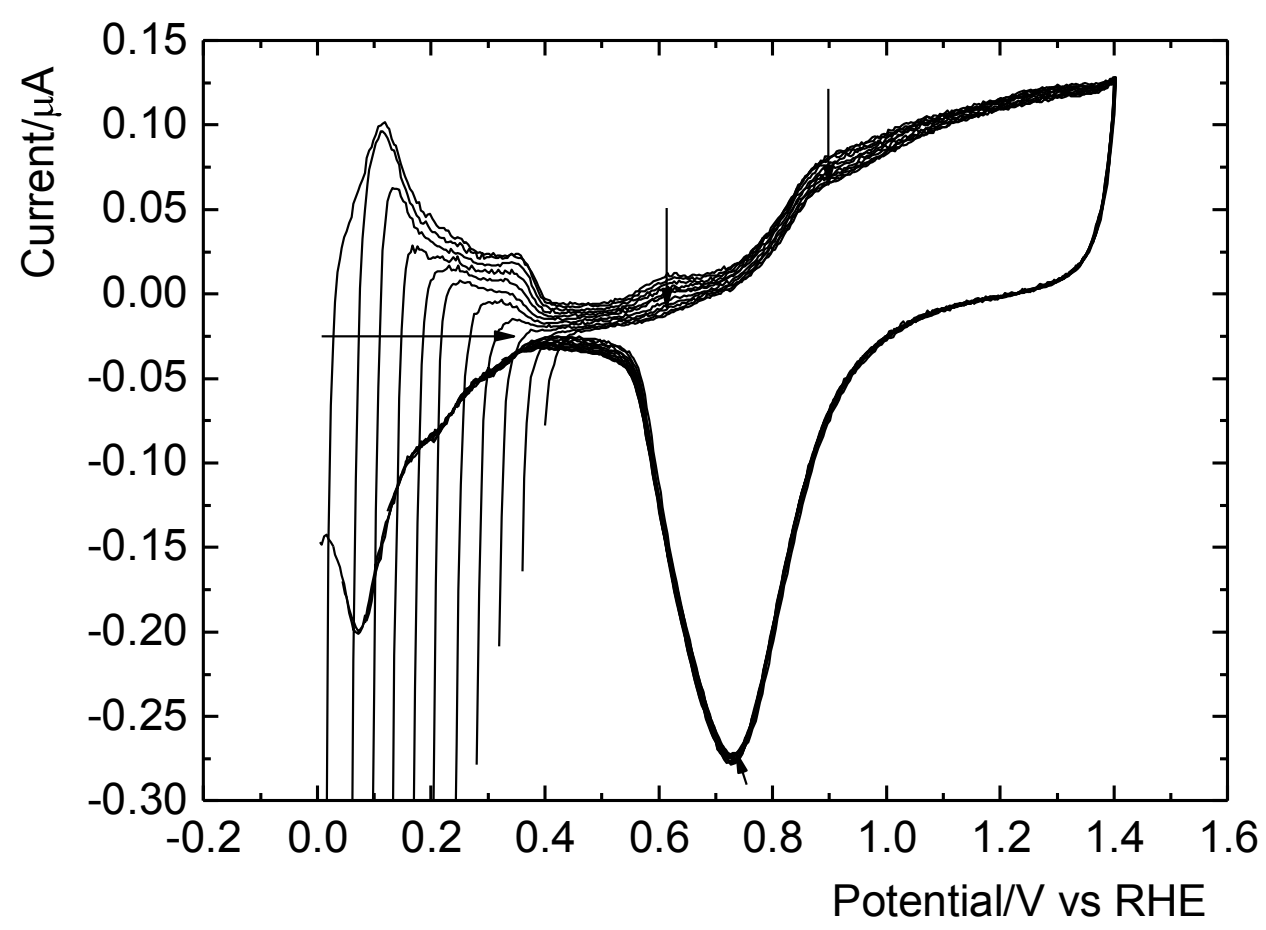

Figure $6 \mathrm{~b}$

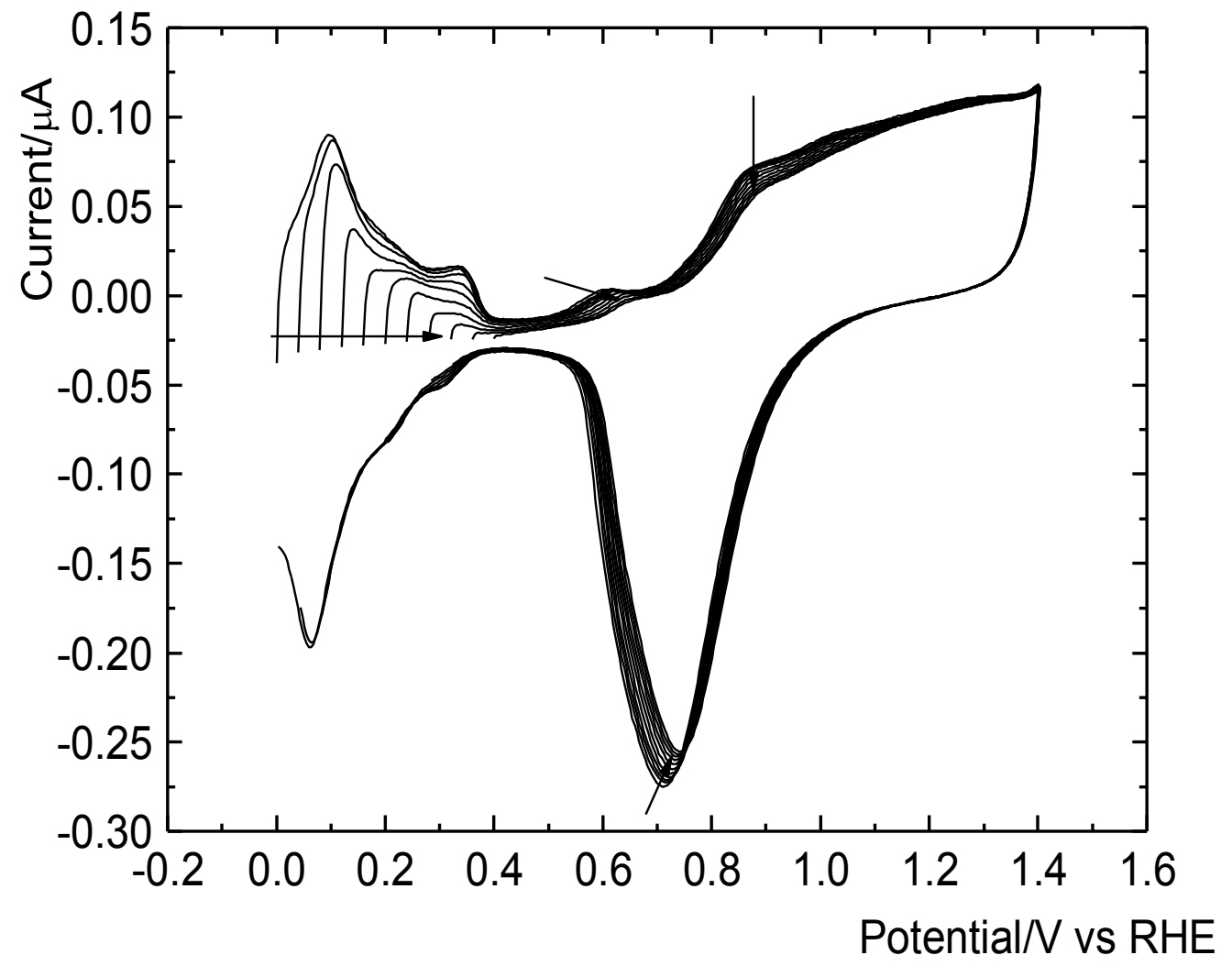


Figure 7

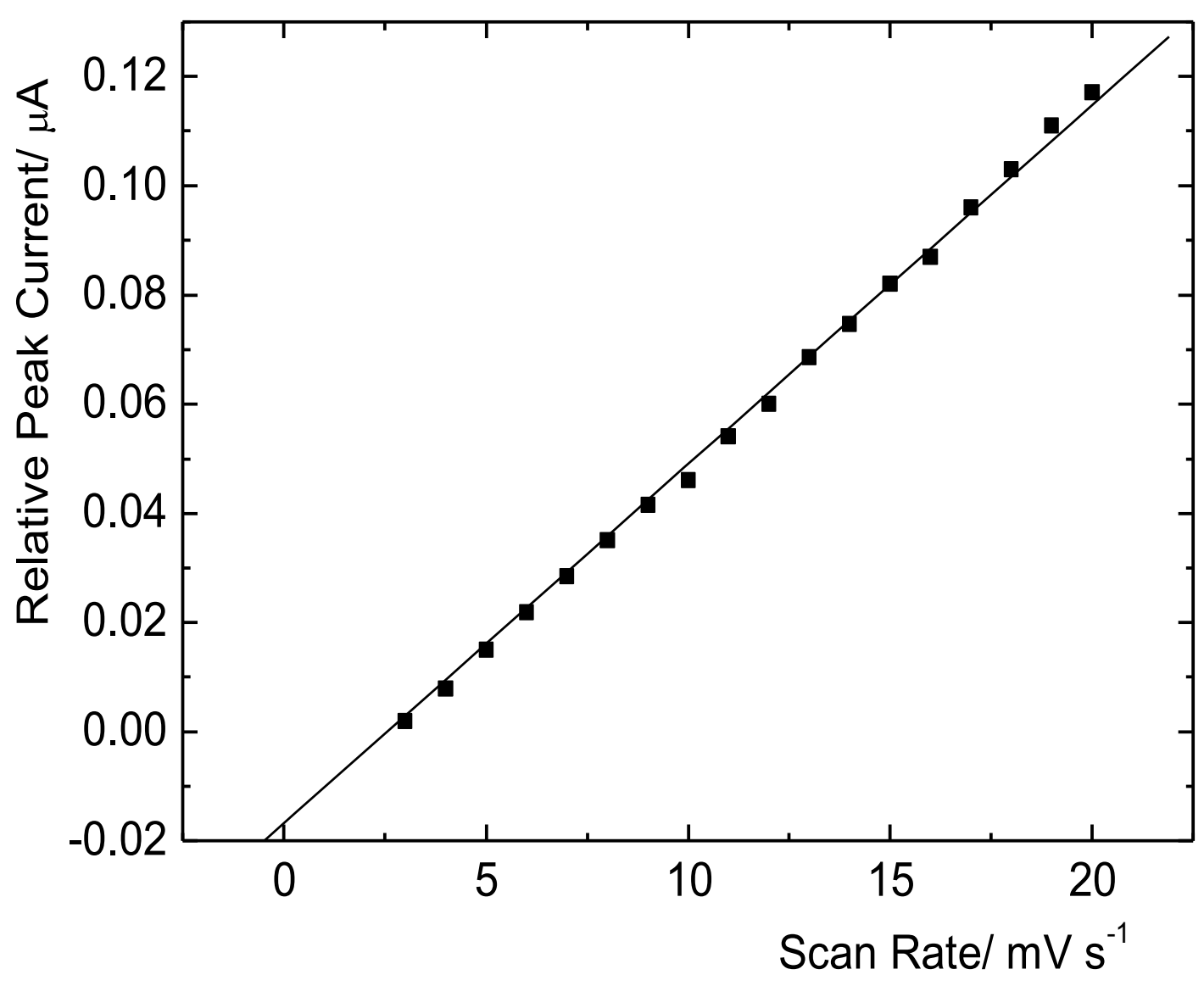

